\title{
Elaboration des réserves protéiques des graines à partir des photosynthétats récents ou remobilisés chez deux variétés de soja. Influence d'irrigations fertilisantes azotées
}

\author{
J. Calmés ${ }^{1}$, N. Gelfi ${ }^{2}$, G. Viala ${ }^{1}$, A. Bouniols ${ }^{2}$ et R. Blanchet ${ }^{2}$ \\ 1 Centre de Physiologie végétale, Unité de Recherche associée au CNRS, $n^{\circ} 241$, Université Paul-Sabatier, 118, route de Narbonne, \\ 31062 Toulouse Cedex; \\ 2 INRA, Station d'Agronomie, Centre de recherche de Toulouse, BP 27, 31326 Castanet-Tolosan, France
}

(reçu le 19-4-1988, accepté le 12-12-1988)

Résumé - Une étude de la photosynthèse et de l'utilisation des assimilats pour l'élaboration des réserves des graines est réalisée pour deux variétés de soja (Glycine max (L.) Merr.), l'une «Weber» à croissance indéterminée, l'autre «172-16» à croissance déterminée. Après des charges en ${ }^{14} \mathrm{CO}_{2}$ effectuées durant la phase végétative, le devenir des composés marqués est suivi durant la période de fructification. Leur utilisation pour le développement des graines est comparée à celle des photosynthétats de formation récente. Ces derniers contribuent surtout à la mise en place des réserves glucidiques et lipidiques tandis que les premiers participent, pour une large part, à l'élaboration des protéines.

La physiologie des deux variétés est très comparable; seule la distribution des assimilats dans la plante varie en fonction du type de croissance. D'un point de vue agronomique, le type déterminé conviendrait mieux pour des cultures intensives. L'irrigation fertilisante durant la fructification, sans modifier fondamentalement la formation et le devenir des photosynthétats, réduit le rôle du métabolisme azoté des feuilles dans l'alimentation des graines. Elle stimule l'activité photosynthétique; elle pourrait compléter une insuffisance de réserves azotées du sol et remédier à une fixation symbiotique déficiente.

soja - transfert - remobilisation - protéogenèse des graines - irrigation fertilisante

Summary - Storage in the seeds of newly synthetized or remobilized assimilates on two soybean cultivars: nitrogen fertilizing irrigation efficiency. Photosynthesis and storage in the seeds of soybean (Glycine max (L.) Merr) were studied in two cultivars, an indeterminate genotype "Weber» and a determinate line "172-16". After leaf ${ }^{14} \mathrm{CO}_{2}$ incorporation during the vegetative growth, labelled photosynthates were followed during fructification. They were utilized for pod development, preferentially for seed proteogenesis, while newly synthetized assimilates contributed more to lipidogenesis and glucogenesis. Photosynthate translocation and utilization were similar for both genotypes, only distribution in the whole plant was different. Agronomically, the determinate genotype appears preferable for intensive cropping. Fertilizing irrigation during fructification does not essentially change formation and transfer of assimilates, but foliar nitrogen metabolism contributes to a lesser extent to seed proteogenesis; then photosynthesis is stimulated. A deficiency of the symbiotic fixation and small nitrogen soil reserves can be compensated by fertillizing irrigation.

soybean - translocation - remobilisation - seed proteogenesis - fertilizing irrigation

\section{Introduction}

Les réserves protéiques des graines de soja représentent plus de $40 \%$ de leur matière sèche (Salado-Navarro et al., 1985). La mise en place de ces réserves nécessite l'apport d'une quantité importante d'amides et d'acides aminés, synthéti- sés dans la feuille, au cours du métabolisme photosynthétique (Lea et Miflin, 1980; Latché et al., 1986; Grima-Pettenati et al., 1987). Durant la période végétative, le limbe s'enrichit en composés protéiques : la protéine la plus abondante est la ribulose-bisphosphate carboxylase dont le rôle essentiel est la fixation du $\mathrm{CO}_{2}$ atmosphé- 
rique, mais qui peut également être considérée comme protéine de réserve (Wittenbach et al., 1980; Grima-Pettenati, 1985).

Durant le remplissage des gousses, les acides aminés foliaires disponibles pour les organes receveurs proviennent soit directement de l'activité photosynthétique et de l'absorption tardive d'azote, soit de la dégradation des protéines accumulées dans le limbe au cours de la période végétative (Calmés et Viala, 1987). Parmi les amides et acides aminés transférés vers les graines au cours de leur croissance, il est difficile d'apprécier la part qui relève de la photosynthèse récente de celle qui provient de la protéolyse.

Dans ce travail, des incorporations de ${ }^{14} \mathrm{CO}_{2}$, réalisées à deux stades du développement de la plante, permettent de marquer les composés foliaires synthétisés en période de floraison ou de remplissage des graines. Le devenir des assimilats solubles et insolubles, leurs transferts vers les organes receveurs et leur contribution à l'alimentation des gousses sont étudiés chez deux variétés de soja, différant par leur type de croissance. L'influence d'un apport échelonné d'azote à partir du stade floraison est également précisée. Cet apport, réalisé par irrigations fertilisantes, évite que la fixation symbiotique ne soit fortement handicapée par l'abondance du nitrate dans le sol (Bouniols et al., 1985, 1986).

\section{Matériel et Méthodes}

\section{Matériel végétal et conditions de culture}

L'expérimentation est conduite sur deux variétés de groupe de précocité I, l'une à croissance indéterminée "Weber", lautre de type déterminé "172-16", lignée en cours de sélection à l'INRA de Montpellier. Les deux variétés, semées le 16 avril, sont cultivées au champ en parcelies de $30 \mathrm{~m}^{2}$ avec un peuplement de 60 plantes. $\mathrm{m}^{-2}$. Le sol brun, limono-argileux, de profondeur $0,7 \mathrm{~m}$, contenait, le 4 avril, $60 \mathrm{~kg}$. ha-1 d'azote minéral provenant de reliquats de cultures antérieures. II est bien pourvu en phosphore et potassium. L'inoculation est réalisée avant semis par Bradyrhizobium japonicum, souche G 49; les plantes sont abondamment nodulées.

Durant la période de fructification, les parcelles traitées reçoivent $150 \mathrm{~kg}$.ha-1 d'azote sous forme d'urée ajoutée à l'eau d'arrosage au cours de 3 interventions successives apportant chacune $50 \mathrm{~kg}$ de N. Ces arrosages sont effectués durant la floraison les 2,9 et 17 juillet pour l'irrigation appelée "précoce" et pendant la formation des gousses les 6,20 juillet et 4 août pour l'irrigation appelée "tardive". Les parcelles témoins ont reçu des irrigations non fertilisantes. Dans tous les cas, l'eau fournie satisfaisait sensiblement les besoins de l'évapotranspiration maximale. Les plantes ont atteint la maturité physiologique le 25 septembre.

\section{Techniques utilisées}

Les mesures de photosynthèse nette du couvert sont réalisées selon la méthode de Wells et al. (1982), en caisson transparent à couvercle amovible couvrant $0,5 \mathrm{~m}^{2}$, dans lequel l'air maintenu en une légère surpression est constamment renouvelé. La quantité de $\mathrm{CO}_{2}$ fixé est déterminée d'après la différence des teneurs de l'air, à l'entrée et à la sortie du caisson, et le débit gazeux. Ces mesures sont effectuées en fin de matinée ensoleillée.

Les incorporations de ${ }^{14} \mathrm{CO}_{2}$, réalisées selon une technique précédemment décrite (Calmés et al., 1987a) sont effectuées sur la feuille $\left(F_{g}\right)$ située sur le $9^{\theta}$ nœud à partir du bas de la tige principale. La foliole centrale bien éclairée est placée durant $30 \mathrm{~min}$ dans une enceinte de $400 \mathrm{~cm}^{3}$ où l'on fait dégager $3,7 \mathrm{MBq}$ de ${ }^{14} \mathrm{CO}_{2}$ par action de l'acide lactique sur du bicarbonate de $\mathrm{Na}$ (radioactivité spécifique : 2050 $\left.\mathrm{MBq} \cdot \mathrm{mmole}^{-1}\right)$. Ces incorporations ont été réalisées le 6 juillet (stade $R_{1}$ de Fehr et al., 1971) et le 25 août (stade $R_{6}$ ). Les plantes marquées au ${ }^{14} \mathrm{C}$ le 6 juillet et destinées à être prélevées en août ou septembre ont reçu une charge 4 fois plus élevée ( $14,8 \mathrm{ivi} \mathrm{Bq}$ ).

Les folioles, le pétiole et les gousses du même étage sont prélevés séparément et placés aussitôt dans un congélateur à $-18^{\circ} \mathrm{C}$; pour les expérimentations de longue durée, l'ensemble des parties aériennes a été récolté.

Pour l'analyse des graines, un gramme environ de matériel frais, broyé au Polytron dans $20 \mathrm{ml}$ de $\mathrm{N}$-butanol, est porté à $100^{\circ} \mathrm{C}$ durant $10 \mathrm{~min}$. Les lipides sont solubilisés par ce premier traitement suivi de 2 extractions au mélange chloroforme-méthanol $(2: 1, v / v)$ (Calmés et al., 1987a). Une fois délipidé, le résidu est soumis à 3 extractions aqueuses. Les surnageants recueillis par centrifugation sont mélangés à l'eau de lavage des lipides et concentrés; ils constituent la fraction soluble, analysée ensuite par chromatographie sur résines Dowex 50 et 1 . Les protéines contenues dans le culot résiduel sont hydrolysées par $\mathrm{HCl}, 6 \mathrm{~N}$ et les acides aminés libérés fixés sur résines Dowex 50 . La radioactivité des diverses fractions solubles est mesurée au compteur à scintillation liquide Packard $460 \mathrm{C}$; celle des fractions insolubles est mesurée après combustion au moyen de l'Oxidizer Packard. Pour l'analyse des feuilles et des cosses, la délipidation est inutile et le broyage est effectué directement en milieu aqueux.

\section{Résultats}

Caractéristiques culturales : photosynthèse et productivité

La photosynthèse nette du couvert végétal est mesurée du début juillet (floraison) jusqu'à la mi-septembre (fin du remplissage des graines). Pour la variété "Weber", à croissance indéterminée, l'intensité de la photosynthèse présente des variations importantes (Fig. 1A). C'est vers la mi-juillet, lorsque la croissance de l'appareil végétatif est très active, que la parcelle témoin assimile le maximum de $\mathrm{CO}_{2}$ (près de $20 \mu$ moles. $\mathrm{m}^{-2} \cdot \mathrm{s}^{-1}$ ). Au début du mois d'août, le 
A

$172-16$
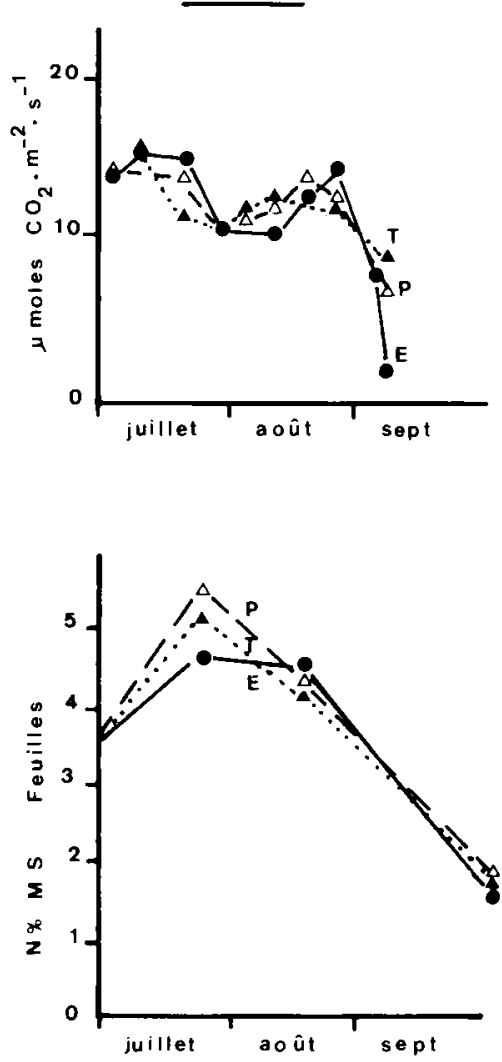

\section{WEBER}

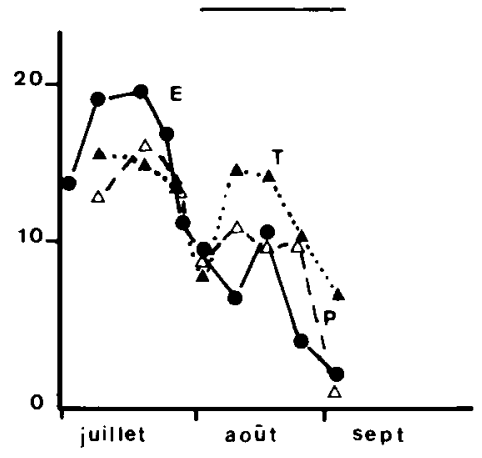

B

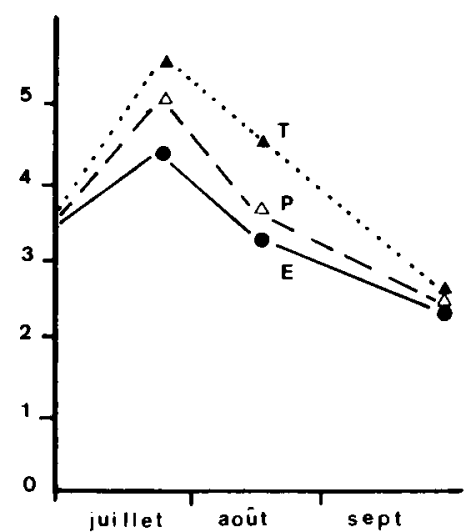

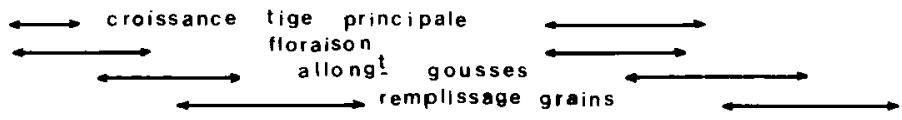

Fig. 1. A. Evolution, à partir de la floraison, de la photosynthèse nette du couvert mesurée à l'aide de caissons transparents couvrant $0,5 \mathrm{~m}^{2}$ de sol. B. Evolution de la teneur des feuilles en azote total (en $\%$ de la masse de matière sèche) au cours de la fructification. - $E$, irrigation eau; irrigations eau $+N: \Delta P$, précoce, $\Delta \mathrm{T}$, tardive. Ecart type des mesures : très voisin de $10 \%$ des valeurs moyennes.

développement de la tige principale se termine; l'assimilation devient environ trois frois plus faible; après une augmentation pendant la phase du remplissage des graines, elle décroît rapidement. L'irrigation fertilisante ne modifie pas fondamentalement cette évolution, sauf en ce qui concerne l'apport tardif d'azote qui se traduit par une reprise importante de l'activité photosynthétique durant le mois d'août.

En revanche, la variété «172-16", à croissance déterminée, présente des valeurs de photosynthèse beaucoup plus stables : elles se situent aux environs de $12 \mu$ moles. $\mathrm{m}^{-2} . \mathrm{s}^{-1}$. On observe cependant une baisse momentanée de ces valeurs au début du mois d'août, avant l'intense développement des graines. La reprise qui fait suite à ce fléchissement se produit plus tôt lorsqu'il y a irrigation fertilisante.

Les rendements importants obtenus en l'ab- sence d'apport d'azote (Tableau I) témoignent d'une bonne fixation symbiotique. Toutefois les plantes réagissent à l'irrigation fertilisante qui se traduit, au moins en juillet et au début août, par une augmentation de la teneur des feuilles en azote total (Fig. 1B). La valeur maximale, qui se situe à la fin de la floraison et au début de la formation des gousses, croît de 18\% par rapport au témoin pour le cultivar " $172-16$ " et de $26 \%$ pour «Weber». Le cultivar déterminé, dont les rendements en graines sont généralement supérieurs à ceux du type indéterminé, mobilise mieux l'azote et l'ensemble des assimilats au profit des gousses; l'irrigation fertilisante "tardive» accroît sensiblement la production de graines pour la variété «172-16». En revanche, avec la variété "Weber", l'irrigation fertilisante est sans grand bénéfice pour la fructification. 
Tableau I. Influence de l'irrigation fertilisante sur les principaux caractères des deux types variétaux à maturité. Moyenne de 14 plantes \pm écart type.

\begin{tabular}{|c|c|c|c|c|c|c|}
\hline \multirow{3}{*}{$\begin{array}{l}\text { Cultivar } \\
\text { Irrigation }\end{array}$} & \multicolumn{3}{|c|}{$172-16$} & \multicolumn{3}{|c|}{ Weber } \\
\hline & \multirow[t]{2}{*}{ Eau } & \multicolumn{2}{|c|}{ fertilisante } & \multirow[t]{2}{*}{ Eau } & \multicolumn{2}{|c|}{ fertilisante } \\
\hline & & Précoce & Tardive & & Précoce & Tardive \\
\hline Plante & & & & & & \\
\hline Hauteur $(\mathrm{cm})$ & $53 \pm 7$ & $56 \pm 14$ & $47 \pm 9$ & $107 \pm 35$ & $135 \pm 21$ & $134 \pm 27$ \\
\hline Matière sèche $\left(\mathrm{g} \cdot \mathrm{m}^{-2}\right)$ & $929 \pm 120$ & $933 \pm 70$ & $1068 \pm 110$ & $948 \pm 130$ & $1108 \pm 150$ & $1017 \pm 90$ \\
\hline $\begin{array}{l}\mathrm{N} \text { absorbé }\left(\mathrm{g} \cdot \mathrm{m}^{-2}\right) \\
\text { Graines }\end{array}$ & $35,1 \pm 3,5$ & $36,0 \pm 3,6$ & $40,7 \pm 4,1$ & $32,0 \pm \quad 3,2$ & $36,5 \pm 3,7$ & $37,6 \pm 3,8$ \\
\hline Matière sèche $\left(\mathrm{g} \cdot \mathrm{m}^{-2}\right)$ & $429 \pm 54$ & $443 \pm 31$ & $504 \pm 16$ & $402 \pm 55$ & $418 \pm 32$ & $435 \pm 11$ \\
\hline Protéines (\%) & $43,3 \pm 0,6$ & $42,0 \pm 0,6$ & $42,0 \pm 0,6$ & $38,7 \pm 0,5$ & $40,6 \pm 0,5$ & $40,6 \pm 0,5$ \\
\hline Lipides (\%) & $19,9 \pm 0,5$ & $20,5 \pm 0,5$ & $20,7 \pm 0,5$ & $21,5 \pm 0,4$ & $21,4 \pm 0,4$ & $21,3 \pm 0,4$ \\
\hline $\mathrm{N}$ graines/ $\mathrm{N}$ absorbé & 0,85 & 0,83 & 0,83 & 0,78 & 0,74 & 0,75 \\
\hline Indice de récolte & 0,46 & 0,47 & 0,47 & 0,42 & 0,38 & 0,43 \\
\hline
\end{tabular}

\section{Transferts et devenir des assimilats foliaires}

Distribution dans la plante, au stade croissance des graines, des assimilats formés au début de la floraison

Après incorporation foliaire de ${ }^{14} \mathrm{CO}_{2}$ au début de la floraison ( 6 juillet: stade $R_{1}$ ), la distribution de la radioactivité dans la plante a été étudiée, 51 jours plus tard, durant la croissance des graines (stade $R_{6}$ ). La Figure 2 indique la répartition (\%) du radiocarbone et de la masse de matière sèche des feuilles et des gousses regroupées en plusieurs ensembles suivant leur niveau d'insertion sur la tige. Pour la variété «172-16", les ramifications, qui représentent plus de la moitié de la masse totale de la plante, reçoivent beaucoup de radiocarbone. Les gousses portées directement par la tige principale présentent un

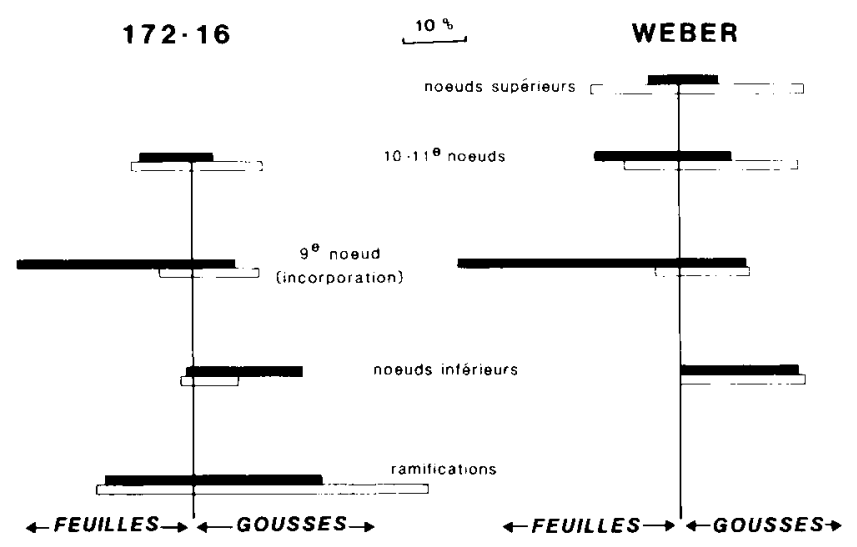

Fig. 2. Répartition du radiocarbone $\square$ et de la matière sèche $\square$ entre les feuilles (folioles + pétioles) et les gousses (cosses + graines), regroupées en plusieurs ensembles suivant leur niveau d'insertion sur la tige. Feuilies + gousses $=100$. Charge en ${ }^{14} \mathrm{CO}_{2}$ effectuée sur la $9^{e}$ feuille à partir du bas, le 6 juillet; prélèvement 51 jours plus tard ( 26 août). marquage d'autant plus intense qu'elles se trouvent en position basse.

Dans les conditions de peuplement réalisées, les pieds de la variété "Weber" n'ont pas de ramifications. En revanche, les feuilles et les fructifications situées au-dessus du ge nœud (zone d'incorporation) sont beaucoup plus nombreuses que pour le type déterminé et drainent davantage d'assimilats. Toutefois, le marquage spécifique des gousses (radioactivité par rapport à la masse de matière sèche) s'avère, comme pour la variété "172-16", d'autant plus grand qu'elles s'insèrent vers le bas de la tige.

Devenir des assimilats foliaires formés au début de la floraison et transferts vers les graines

Une série de charges folaires en ${ }^{14} \mathrm{CO}_{2}$ est réalisée le 6 juillet. Un premier prélèvement est effectué $30 \mathrm{~h}$ plus tard, les autres sont échelonnés durant le remplissage des graines, 51,59 ou 66 jours après incorporation. Dans la foliole, l'évolution de la répartition du carbone 14 entre les composés azotés (protéines et acides aminés libres) et glucidiques (solubles et insolubles) est indiquée sur la Figure 3. La radioactivité globale des deux catégories de métabolites diminue progressivement; cette diminution apparaît plus nettement pour les composés azotés, surtout lors des deux derniers prélèvements effectués à l'époque où le remplissage des graines est intense. Les résultats obtenus sont très comparables pour les deux variétés «172-16" et «Weber» : les valeurs indiquées sur la figure se rapportent à la première.

Corrélativement, dans les graines, le radiocarbone s'incorpore dans les diverses catégories de substances mises en réserves (Fig. 3 ). Le marquage de la fraction protéique prédomine 


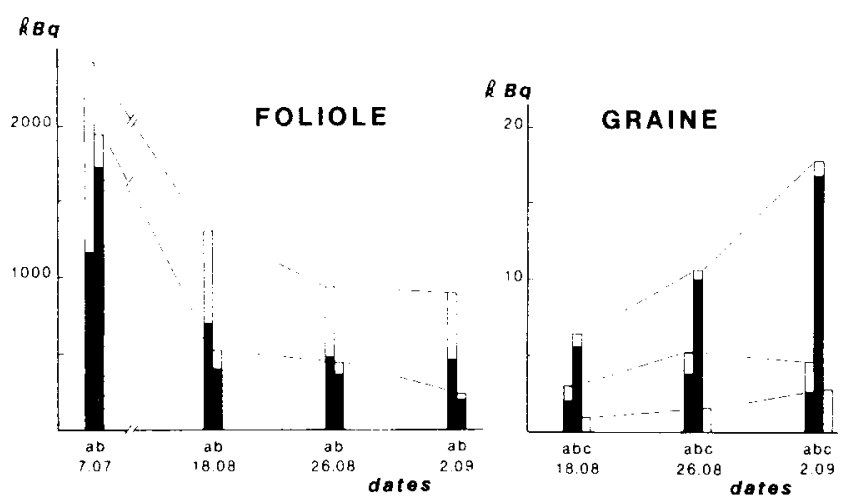

Fig. 3. Transfert de la radioactivité $(\mathrm{kBq})$ des composés de la foliole centrale vers une graine du même étage après une incorporation foliaire en ${ }^{14} \mathrm{CO}_{2}$ effectuée le 6 juillet. Variété "172-16". a, composés glucidiques et acides organiques : - hydrosolubles, insolubles; b, composés azotés : $\square$ acides aminés libres, protéines; c, lipides.

toujours; il s'accentue même en fin de remplissage, où la radioactivité des composés azotés représente $72 \%$ de l'ensemble du radiocarbone des graines.

Comparaison de l'utilisation des assimilats néoformés et des assimilats stockés depuis le début de la floraison

Cette comparaison, effectuée le 26 août, durant la période de remplissage des graines, concerne les plantes qui ont subi une charge foliaire en ${ }^{14} \mathrm{CO}_{2}$, les unes 51 jours auparavant, les autres simplement $30 \mathrm{~h}$ plus tôt. Le marquage des composés solubles (glucides, amides et acides aminés libres), susceptibles de transfert vers les organes receveurs, est étudié dans le limbe, le pétiole, les cosses et les graines de l'étage traité (Tableau II). Pour les assimilats formés au début de la floraison, le rapport de la radioactivité des amides et des acides aminés libres sur celle des glucides solubles présente une valeur nettement supérieure à celle des assimilats néoformés, aussi bien dans les cosses que dans les graines et, à un degré moindre, dans le pétiole et la foliole. Ainsi, l'étude de la fraction soluble montre que le marquage des composés azotés est proportionnellement plus important lorsque l'incorporation de ${ }^{14} \mathrm{CO}_{2}$ date du début floraison. Ceci s'observe avec les deux variétés : «172-16» et «Weber".

Le marquage important des composés azotés se retrouve lorsqu'on analyse l'ensemble des constituants solubles et insolubles de la graine : 51 jours après l'incorporation réalisée au début de la floraison, les composés azotés sont les plus radioactifs (Fig. 4); viennent ensuite les glucides totaux, puis les lipides. Ainsi, dans les graines de "Weber", ces trois catégories de constituants renferment respectivement 65,26 et $9 \%$ du radiocarbone. En revanche, après une incorporation effectuée au cours du remplissage des graines, on observe, $30 \mathrm{~h}$ plus tard, dans les organes receveurs, une distribution inverse du

Tableau II. Radiocarbone ( $\mathrm{kBq}$ par organe) des glucides hydrosolubles et des acides aminés libres (amides comprises) dans les organes correspondant à l'étage traité. Comparaison des deux variétés pour des incorporations foliaires de ${ }^{14} \mathrm{CO}_{2}$ effectuées à deux stades de développement. Moyenne de 6 échantillons. Ecart type des mesures : très voisin de $5 \%$ des valeurs moyennes.

Foliole Pétiole Cosses Graines

Charge 6-07, récolte 51 jours après (26-08)

Variété «172-16"

Glucides hydrosolubles

Acides aminés libres

Acides aminés/glucides

Variété "Weber"

Glucides hydrosolubles

Acides aminés libres

Acides aminés/glucides

Charge 25-08, récolte $30 \mathrm{~h}$ après

Variété «172-16»

Glucides hydrosolubles

Acides aminés libres

Acides aminés/glucides

Variété "Weber"

Glucides hydrosolubles

Acides aminés libres

Acides aminés/glucides

$\begin{array}{cccc}295 & 52,1 & 5,6 & 3,9 \\ 62 & 6,6 & 1,8 & 3,4 \\ (0,21) & (0,13) & (0,32) & (0,87) \\ & & & \\ 259 & 38,5 & 4,1 & 3,5 \\ 55 & 7,8 & 2,3 & 3,8 \\ (0,21) & (0,20) & (0,56) & (1,09)\end{array}$

252

36

$(0,14)$

180

36

$(0,20)$
41,1

5,0

$(0,12)$

38,4

4,4

$(0,11)$
18,8

2,1

$(0,11)$

31,5

19,2

2,3

30,3

$(0,12)$
4,5

$(0,15)$ 

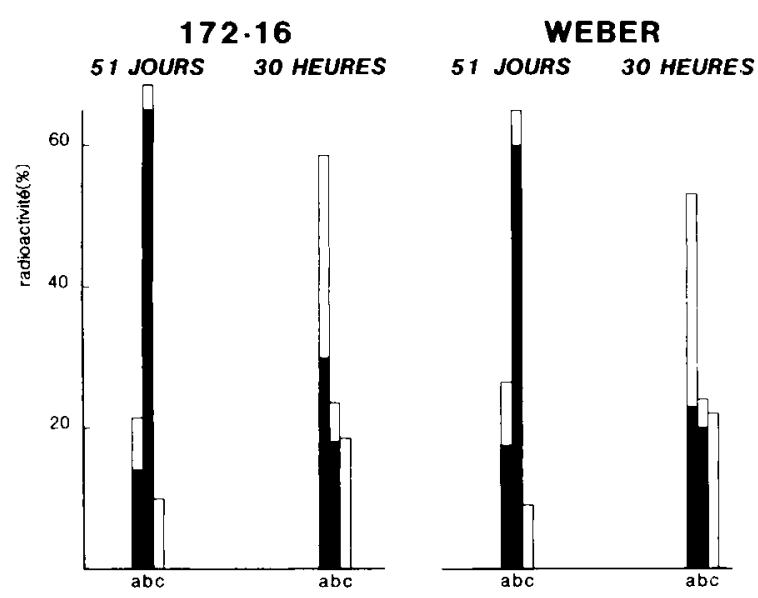

Fig. 4. Distribution (\%) du radiocarbone dans les graines entre les composés glucidiques, azotés et lipidiques (mêne légende que pour la Figure 3). Moyenne de 6 échantillons. Charge foliaire en ${ }^{14} \mathrm{CO}_{2}$ effectuée soit le 6 juillet avec prélèvement 51 jours plus tard, soit le 25 août avec prélèvement $30 \mathrm{~h}$ plus tard.

radiocarbone : le marquage des glucides totaux prédomine largement sur celui des substances azotées. Les résultats obtenus avec les deux variétés sont comparables (Fig. 4).

L'ensemble de ces données traduit une utilisation différente du carbone pour la mise en place des réserves de la graine selon l'époque où il est assimilé (floraison ou remplissage des graines).

\section{Influence des irrigations fertilisantes}

Les irrigations fertilisantes ne modifient en aucun cas la composition des graines ni la distribution du radiocarbone entre ses constituants (Fig. 5A.). De plus, cette distribution est sensiblement la même avec les deux variétés (Fig. 5B) pour lesquelles le marquage des composés solubles foliaires est très comparable (Tableau II).

Les valeurs moyennes obtenues à partir des deux variétés (Tableau III) permettent de comparer la radioactivité des composés solubles de plantes bénéficiant d'irrigations fertilisantes, par référence à des cultures témoins arrosées avec de l'eau. Comme précédemment, le rapport de la radioactivité des amides + acides aminés libres sur celle des glucides a été établi. L'apport d'azote par irrigation modifie les valeurs de ce rapport: elles deviennent plus faibles dans les folioles et les pétioles, et, au contraire, plus élevées dans les cosses et les graines.

\section{Discussion}

Au moment de la floraison, la feuille $F_{9}$, qui a fixé le ${ }^{14} \mathrm{CO}_{2}$, se trouve en position haute; peu de feuilles en voie de développement se situent au-
A

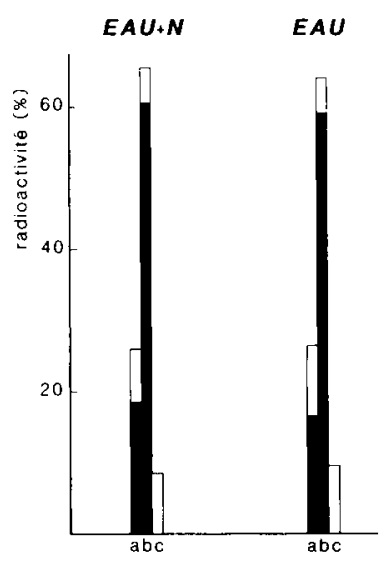

B

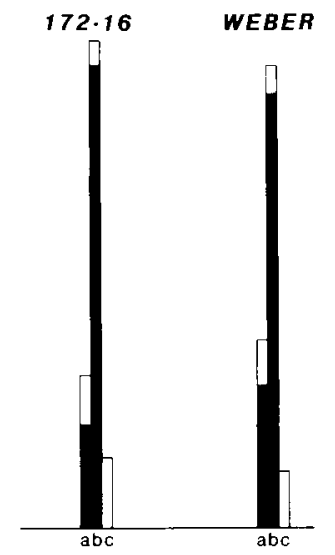

Fig. 5. Distribution (\%) du radiocarbone dans les graines (même légende que pour la Figure 3). Moyenne de 6 échantillons. Les charges en ${ }^{14} \mathrm{CO}_{2}$ sont effectuées le 6 juillet, les prélèvements 51 jours plus tard (26 août). $A$, influence des irrigations fertilisantes; $\mathrm{B}$, comparaison des variétés «17216 " et «Weber».

dessus d'elle dans le cas de la variété «172-16» à croissance déterminée. Chez la variété "Weber", la croissance apicale se poursuit plus longtemps et, peu à peu, la $F_{9}$ n'est plus en position haute. Dans un premier temps, les assimilats marqués contribuent au développement des jeunes feuilles qui se localisent surtout sur les ramifications pour le type déterminé et dans la région apicale pour le type indéterminé. Dans un second temps, les gousses exercent un effet puits, qui augmente au fur et à mesure du remplissage des graines. II en résulte une mobilisation croissante des assimilats foliaires (Thorne et Koller, 1974; Gent, 1982; Hanson et Kenny, 1985; Calmés et al., 1987b). La redistribution du radiocarbone assimilé au stade floraison se fait au profit de toutes les gousses, plus spécialement de celles qui se situent vers le bas de la tige. Cette distribution préférentielle pourrait compenser, en partie, le fait que les feuilles du bas deviennent sénescentes les premières et alimentent de moins en moins leurs propres gousses. En revanche, en période de remplissage des graines, l'activité photosynthétique d'une feuille bénéficie avant tout aux fructifications insérées à son aisselle (Calmés et Viala, 1981).

L'effet puits des gousses se traduit également par une stimulation de l'activité photosynthétique au cours du mois d'août (Fig. 1A); cette stimulation constatée à maintes reprises au cours des années précédentes (Calmés et al., 1987a, b) est nettement favorisée par les irrigations fertilisantes, surtout en ce qui concerne la variété "Weber», pour laquelle la fertilisation entraîne un accroissement sensible de la teneur en azote des feuilles. 
Tableau III. Influence de l'irrigation fertilisante sur le marquage ( $\mathrm{kBq}$ par organe) des glucides hydrosolubles et des acides aminés libres (amides comprises) dans les organes correspondant à l'étage traité. Incorporations foliaires de ${ }^{14} \mathrm{CO}_{2}$ effectuées à deux stades de développement. Moyenne de 6 échantillons. Ecart type des mesures : très voisin de $5 \%$ des valeurs moyennes.

Foliole Pétiole Cosses Graines

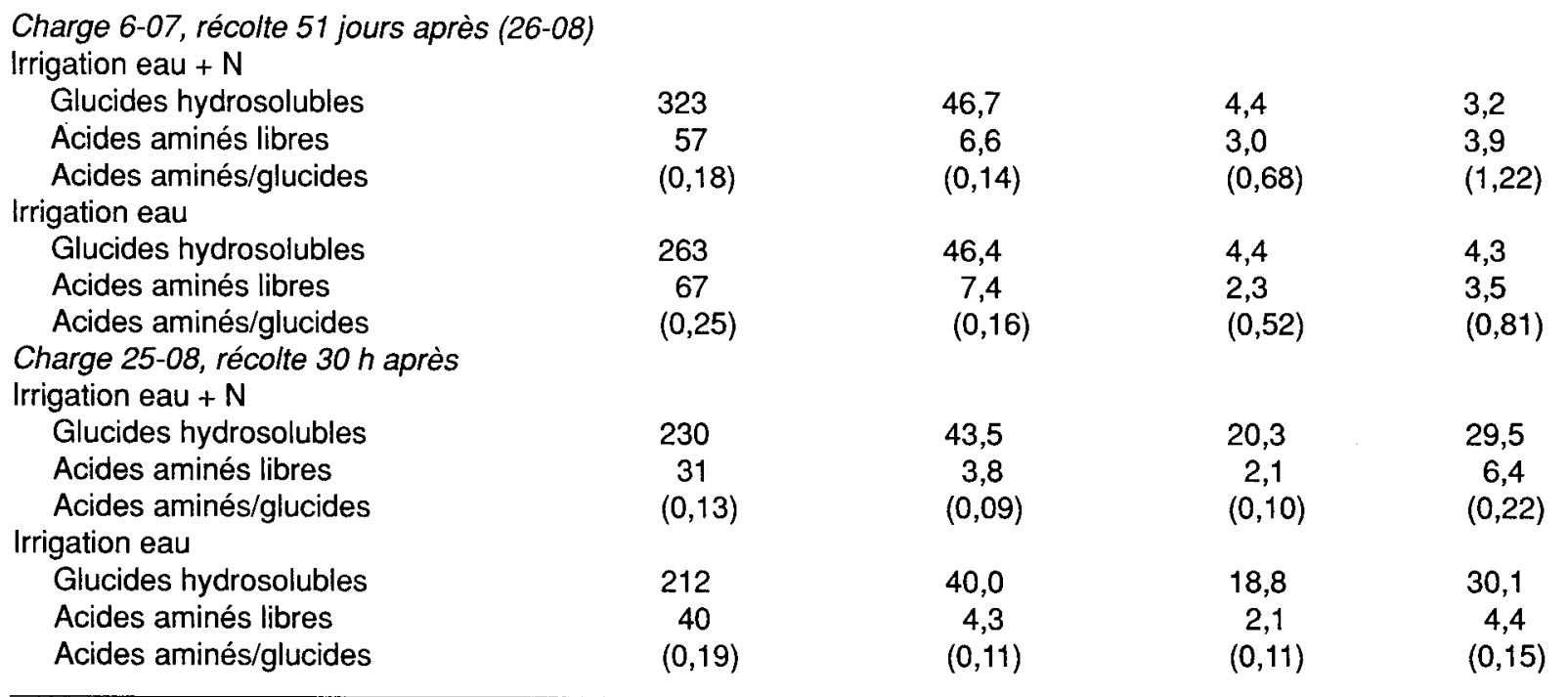

Pour satisfaire à la demande importante d'azote nécessaire à la croissance des graines (Warembourg et al., 1982; Kock et Schrader, 1984; Crafts-Brandner et al., 1984; Warembourg et Fernandez, 1985), la plante mobilise les assimilats synthétisés et stockés dans la feuille au cours de la période végétative et de la floraison. II en résulte un appauvrissement progressif du limbe en azote total (Fig. 1B). Après incorporation foliaire de ${ }^{14} \mathrm{CO}_{2}$ au début de la floraison, on retrouve, au cours du remplissage des graines, la présence, dans le pétiole et les cosses, d'amides et d'acides aminés libres marqués dont la radioactivité est relativement élevée par rapport à celle des glucides solubles. Cette présence témoigne du transfert, sans remaniement complet au niveau des chaînons carbonés, de l'azote aminé foliaire vers les graines. Sur $100 \mathrm{~Bq}$ accumulés dans ces organes puits, 62 au début du remplissage et 72 à la fin (67 en moyenne) le sont sous forme de composés azotés. On sait d'ailleurs que la graine conserve encore l'aptitude à utiliser les amides, alors qu'elle a cessé de métaboliser le saccharose (Vernooy et al., 1986). Ainsi le carbone assimilé par photosynthèse au début de la floraison participe surtout à la mise en place des réserves protéiques.

En revanche, pour le carbone assimilé par la feuille en période de croissance des graines, la fraction qui est transtérée vers ces dernières n'est utilisée que dans la proportion de $24 \%$ pour la formation des réserves azotées. Cette valeur, mesurée $30 \mathrm{~h}$ après l'incorporation, est en accord avec des résultats antérieurs (Calmés et al., 1987b) où il a été montré qu'elle reste sensiblement constante au moins jusqu'à 72h. On peut donc considérer qu'elle représente la part de carbone néoassimilé intégrée dans les protéines.

Durant la période de croissance et jusqu'à maturité, les graines ont des teneurs en protéines qui se situent aux environs de $42 \%$ de leur matière sèche (Calmés et al., 1987b); dans la mise en place de ces protéines, deux apports d'assimilats interviennent simultanément : l'un, dû à la remobilisation, se répartit pour $67 \%$ dans les composés azotés de la graine, l'autre, dû à la photosynthèse récente, pour seulement $24 \%$. Dans la mesure où ces deux proportions se maintiennent jusqu'à maturité, un calcul simple montre que l'apport lié à la remobilisation fournit plus de $40 \%$ des protéines des graines.

Si l'irrigation fertilisante a un effet bénéfique sur le rendement et l'activité photosynthétique, son influence sur le métabolisme et le transfert des assimilats est plus difficile à saisir. On constate toutefois que l'apport d'azote se traduit, dans les folioles et le pétiole, par un marquage plus faible des acides aminés libres par rapport aux glucides solubles. Ceci s'observe aussi bien avec les photosynthétats néoformés qu'avec les assimilats stockés durant la phase végétative. En 
revanche, dans les cosses et les graines, l'irrigation fertilisante favorise le marquage des acides aminés. Cette inversion est probablement le fait du métabolisme des gousses. Elles reçoivent directement une part importante de l'azote du sol et, au cours des remaniements biochimiques, les chaînons carbonés d'origine foliaire sont davantage impliqués dans la synthèse d'acides aminés. Ainsi, le métabolisme azoté des feuilles serait relativement moins sollicité pour assurer la nutrition des gousses lorsque la plante bénéficie d'irrigations fertilisantes.

D'un point de vue agronomique, dans de bonnes conditions culturales, les deux types de croissance, déterminée ou indéterminée, ne semblent pas influencer le fonctionnement du couvert et l'utilisation des assimilats. Ceci confirme les observations de Beaver et al. (1985) et Blanchet et al. (1986). Toutefois le type déterminé présente une moindre tendance à l'exubérance des organes végétatifs et à la verse; son fonctionnement photosynthétique est plus régulier (Fig. 1) et il paraît mieux adapté pour des techniques culturales intensives. Dans cette perspective, l'irrigation fertilisante, dont l'efficacité physiologique est démontrée au cours de cefte étude, pourrait compléter une insuffisance de réserves azotées de sols peu profonds ou filtrants et remédier à une fixation symbiotique déficiente.

\section{Références}

Beaver J. S., Cooper R.L. \& Martin R.J. (1985) LIry matter accumulation and seed yield of determinate and indeterminate soybeans. Agron. J. 77, 675-679

Blanchet R., Bouniols A., Constant S., Gelfi N., Marty J.R. \& Puech J. (1986) Biologie et formation du rendement de sojas déterminés et indéterminés, en bonnes conditions culturales. Eurosoya 4, 10-17

Bouniols A., Cleyet-Marel J.C., Crozat Y., Drevon J.J., Hecman M.D., Obaton M., Salsac L., Lagacherie B., Conejero G., Robin P., Wery J. \& Warembourg F. (1986) Nutrition azotée du soja : les deux voies de l'assimilation de l'azote. In: Le Soja, Physiologie de la Plante et Adaptation aux Conditions Françaises. Cetiom, Paris, pp. 38-46

Bouniols A., Puech J., Chalamet A. \& Mondies M. (1985) Influence des conditions d'alimentation hydrique ou azotée à différents stades du développement sur la production des graines et la nutrition azotée du soja. Eurosoya 3, 55-61

Calmés J., Naudy M. \& Viala G. (1983) Protéogenèse des graines de soja, Glycine max (L) Merr. : origine des acides aminés. Agronomie 3, 273-278

Calmés J., Gelfi N., Viala G., Gavalié G. \& Blanchet R. (1987a) Photosynthèse et élaboration des réserves des graines chez divers types de soja à croissance indéterminée, semi-déterminée et déterminée. Agronomie $7,33-40$

Calmés J., Gelfi N., Viala G., Bouniols A., Blanchet R.
\& Cavalié G. (1987b) Influence de l'alimentation en azote sur la participation des feuilles au développement des graines chez le soja. Eurosoya 6, 7-14

Calmés J. \& Viala G. (1981) Métabolisme photorespiratoire : développement et fructification du soja. Physiol. Veg. 19, 503-511

Calmés J. \& Viala G. ( 1987) Relations organes donneurs et organes receveurs chez le soja : migrations vers les graines des composés azotés foliaires. $C . R$. Acad Sci., Paris, série III 305, 165-169

Crafts-Brandner S.J., Below F.E., Harper J.E. \& Hageman R.H. (1984) Effect of nodulation on assimilate remobilization in soybean. Plant Physiol. 76, 452-455

Fehr W.R., Caviness C.E., Burmood D.J. \& Pennington J.S. (1971) Stages of development description for soybean Glycine max. Crop. Sci. 11, 929-931

Gent M.P.N. (1982) Effect of defoliation and depodding on long distance translocation and yield in Y-shaped soybean plants. Crop Sci. 22, 245-250

Grima-Pettenati J. (1985) Effets d'une privation d'azote sur la nutrition carbonée et la dégradation des protéines foliaires chez le soja. Thèse de doctorat de $3^{0}$ cycle, Université Paul Sabatier, Toulouse

Grima-Pettenati J., Bailly-Fenech G. \& Latché J.C. (1987) Etude comparative des migrations d'assimilats chez deux variétés de soja, de type déterminé ou indéterminé. Influence d'une carence en azote. Agronomie 7, 447-456

Hanson W.D. \& Kenny S.T. (1985) Genotypic differences in soybean affecting the rate of assimilate transport from the leaf. Crop Sci. 25, 229-234

Koch K.E. \& Schrader L.E. (1984) ${ }^{14}$ C-photosynthate partitioning and translocation in soybeans during reproductive development. Plant Physiol. 75, 10401043

Latché J.C., Calmés J., Naudy-de-Serres M., Viala G. \& Cavalié G. (1986) Mise en réserve temporaire d'azote sous forme organique dans la tige et les feuilles de soja. Physiol. Vég. 24, 707-717

Lea P.J. \& Miflin B.J. (1980) Transport and metabolism of asparagine and other nitrogen compounds within the plant. In: The Biochemistry of Plants. 5: Amino Acids and Derivates. (Miflin B.J., ed.) Academic Press, New York, London, pp. 569-607

Salado-Navarro L.R., Hinson K. \& Sinclair T.R. (1985) Nitrogen partitioning and dry matter allocation in soybeans with different seed protein concentration. Crop Sci. $25,451-455$

Thorne J.H. \& Koller H.R. (1974) Influence of assimilate demand on photosynthesis, diffusive resistance, translocation and carbohydrate levels of soybean leaves. Plant Physiol. 54, 201-207

Vernooy C.D., Thorne J.H., Lin W. \& Rainbird R.M. (1986) Cessation of assimilate uptake in maturing soybean seeds. Plant Physiol. 82, 222-225

Warembourg F.R. \& Fernandez M.P. (1985) Distribution and remobilization of symbiotically fixed nitrogen in soybean Glycine max. Physiol. Plant. 65, 281-286

Warembourg F.R., Montange D. \& Bardin R. (1982) The simultaneous use of ${ }^{14} \mathrm{CO}_{2}$ and $15 \mathrm{~N}_{2}$ labelling techniques to study the carbon and nitrogen economy of legumes grown under natural conditions. Physiol. Plant. 56, 46-55 
Wells R., Schulze L.L., Ashley D.A., Boerma H.R. \& Brown R.H. (1982) Cultivar differences in canopy apparent photosynthesis and the relationship to seed yield in soybeans. Crop. Sci. 22, 886-890

Wittenbach V.A. (1978) Breakdown of ribulose-bisphosphate carboxylase and changes in proteolytic activity during dark induced senescence of wheat seed- lings. Plant Physiol. 62, 604-608

Wittenbach V.A., Ackerson R.C., Giaquinta R.J. \& Hebert P.R. (1980) Changes in photosynthesis, ribulose-bisphosphate carboxylase, proteolytic activity and ultrastructure of soybean leaves during senescence. Crop. Sci. 20, 225-231 